\title{
Research on Railway Speed-up Scheme Based on Grey Decision Theory
}

\author{
Kexin ZHANG, Jiaying HAN ${ }^{1}$, Xiaotie WANG \\ Railway Science and Technology Research and Development Center, China \\ Academy of Railway Sciences Corporation Limited, Beijing 100081, China
}

\begin{abstract}
In this paper, the decision-making problem of speed-up scheme for existing high-speed railway is studied. Considering the different running speed of different parts of high-speed railway, the speed-up scheme of existing line is formulated. Taking the factors affecting the decision-making of railway speed-up scheme into consideration, the efficiency, cost and influence are determined as the evaluation indexes, and the grey target decision-making method in grey system theory is selected to evaluate each scheme. In this problem, qualitative analysis can be used for the optimal decision-making of the initial scheme. The corresponding situations under each index are comprehensively considered and sorted. The value is assigned to each qualitative evaluation to form the effect vector of each situation. In the spherical grey target formed by grey target decision method, the optimal scheme can be obtained by comparing the off-target distance of each effect vector corresponding to the scheme. It is proved that the grey target decision-making method can also obtain the optimal scheme through qualitative analysis when it is difficult to make quantitative analysis.
\end{abstract}

Keywords. High speed railway, grey target decision-making, theoretical analysis, speed increase of existing lines, grey system theory

\section{Introduction}

Railway transportation is very important to the leap forward development of China's economy. In the mid-1990s, railway transportation showed the phenomenon of insufficient transportation capacity and slow growth of scale, which seriously affected the further expansion of China's economic scale. The slow running speed of railway trains has become the bottleneck of productivity development. Since 1997, China has carried out six large-scale speed-up for ordinary speed railways. The sixth large-scale speed-up of Chinese Railway has achieved a qualitative leap on the basis of the first five [1]. Under the background that China's high-speed railway network has basically take shape and the road network structure is increasingly optimized, social and economic development further puts forward the requirements for high-speed railway speed increase [2]. At present, many literatures have studied the economic significance of high-speed railway speed increase to society [3-5]. However, how to speed up high speed railway, maximize the supply of passenger transport, and further improve the

\footnotetext{
${ }^{1}$ Corresponding Author, Han Jiaying, Railway Science and Technology Research and Development Center, China Academy of Railway Sciences Corporation Limited, Beijing,China; E-mail: 15771363401@163.com
} 
quality and efficiency of China's railway operation, is an important problem we need to solve.

At present, there are few projects related to speed-up of existing high-speed railway lines in China. The speed-up projects of existing high-speed railway carried out in Japan on the Tokaido Shinkansen, Sanyo Shinkansen and Tohoku Shinkansen have greatly improved the quality and efficiency of railway operation. Compared with the newly-built high-speed railway, the speed-up of some existing high-speed railways with large passenger flow and good line conditions has the advantages of less investment and quick effect, and can adapt to the needs of current passenger transport as soon as possible, give full play to the scale effect of road network, so as to maximize the benefits.

The selection of railway speed-up scheme is an optimization problem through scientific mathematical tools under the consideration of various comprehensive indexes. In this paper, the grey target decision-making method in grey system theory is used to qualitatively analyze the existing high-speed railway speed-up scheme, and the comprehensive evaluation system and optimization method of the scheme are studied.

\section{Establishment of Evaluation Index for Speed up Scheme}

The decision-making problem of railway speed-up scheme is essentially a multi-objective optimization decision-making problem. There are many researches on multi-objective optimization decision-making problems in China and abroad. For example, the utility function theory of fuzzy multi-attribute group decision-making, the comprehensive decision-making method of the center of gravity of fuzzy set and the scheme decision-making method of grey correlation degree are used to establish a model to evaluate the relevant schemes after obtaining the relevant evaluation indexes [6-10]. Grey decision-making is carried out when the decision-making model contains grey elements or the general decision-making model is combined with the grey decision-making model. It is mainly It is mainly applicable to the problem of scheme selection.

Different sections of existing high-speed railway run at different speeds according to the limitations of the line, so the speed-up target values are different in sections. The evaluation index of the analysis mainly includes three aspects.

(1) Efficiency, including the influence of speed increase on line carrying capacity and transmission capacity. The influence on line carrying capacity includes: the influence of speed ratio of passenger train to freight train, the change of capacity utilization rate, the influence of deducting speed ratio of different classes of trains, and the elastic change of train working diagram; The influence on line transmission capacity includes: the change of the number of passenger train formation, the change of tractable weight of freight train, the influence of passenger and freight train density, and the occupancy rate of passenger train.

(2) Cost, including investment cost and operation cost. The main items that constitute the investment cost are the reconstruction cost of infrastructure equipment such as line, bridge, tunnel, crossing and signal, and the purchase of rolling stock, etc. The main items that constitute the operating cost are vehicle maintenance cost, energy consumption cost, infrastructure maintenance cost, project-related staff salary expenditure, etc. [11]. 
(3) Impact, including the impact on the transportation capacity of the line and related over-lines, and the impact on the ecological environment caused by the speed-up project.

Due to the diversity of high-speed railway operation environment and the complexity of speed-up project related factors, there are a lot of uncertain factors in the actual implementation process of speed-up project, such as line conditions, management level, weather conditions, equipment operation, etc. These uncertain factors have great influence on the cost and efficiency of speed-up, and the evaluation index of decision-making scheme is difficult to quantify to a certain extent. Therefore, the grey target decision-making model can be used for qualitative analysis and optimal selection of speed-up schemes of existing high-speed railway lines.

\section{Establishment of Scheme Decision Model}

\subsection{Grey Target Decision Theory}

Grey target decision-making method is an important method in grey system theory to solve multi-objective decision-making problem. In the case of no standard model for each evaluation index, the unified dimension mathematical transformation is carried out for each index, so that it can be compared in Euclidean space, which includes all the decision-making schemes, that is, the grey target. In the grey target, a certain vector is taken as the target center, that is, the reference point for comparison. Each scheme obtains its own off-target distance by comparing with the target center, and the priority of the schemes are determined through the comparison of the off-target distance [12].

The four elements of grey target decision method are event, countermeasures, goal and effect. The modeling steps are as follows:

(1) Determine the event set, countermeasure set and situation set.

All events within a certain research scope are the event sets of the research scope, which are recorded as follows:

$$
A=\left\{a_{1}, a_{2}, \ldots a_{n}\right\}
$$

All the corresponding possible countermeasures are called countermeasure sets, which are recorded as follows:

$$
B=\left\{b_{1}, b_{2}, \ldots b_{m}\right\}
$$

Cartesian product of event set $A=\left\{a_{1}, a_{2}, \ldots a_{n}\right\}$ and countermeasure set $B=\left\{b_{1}, b_{2}, \ldots b_{m}\right\}$

$A \times B=\left\{\left(a_{i}, b_{j}\right) \mid a_{i} \in A, b_{j} \in B\right\}$ is a situation set. $\left(a_{i}, b_{j}\right)$ is called situation $s_{i j}$

$\boldsymbol{u}_{i j}=\left(u_{i j}{ }^{(1)}, u_{i j}{ }^{(2)}, \ldots, u_{i j}{ }^{(s)}\right) \in S^{s}$ is the effect vector of the situation $s_{i j}$. 
$u_{i j}{ }^{(k)}(k=1,2, \ldots, s)$ is the effect value of the situation $s_{i j}$ under the target $k$.

(2) Rank all situations qualitatively under each index.

(3) Determine the optimal effect vector according to the optimal effect of sorting results, establish the grey target model.

(4) Calculate the off-target distance of each situation effect vector based on grey target, and evaluate the schemes.

The flow chart of grey target decision algorithm is shown in Figure 1

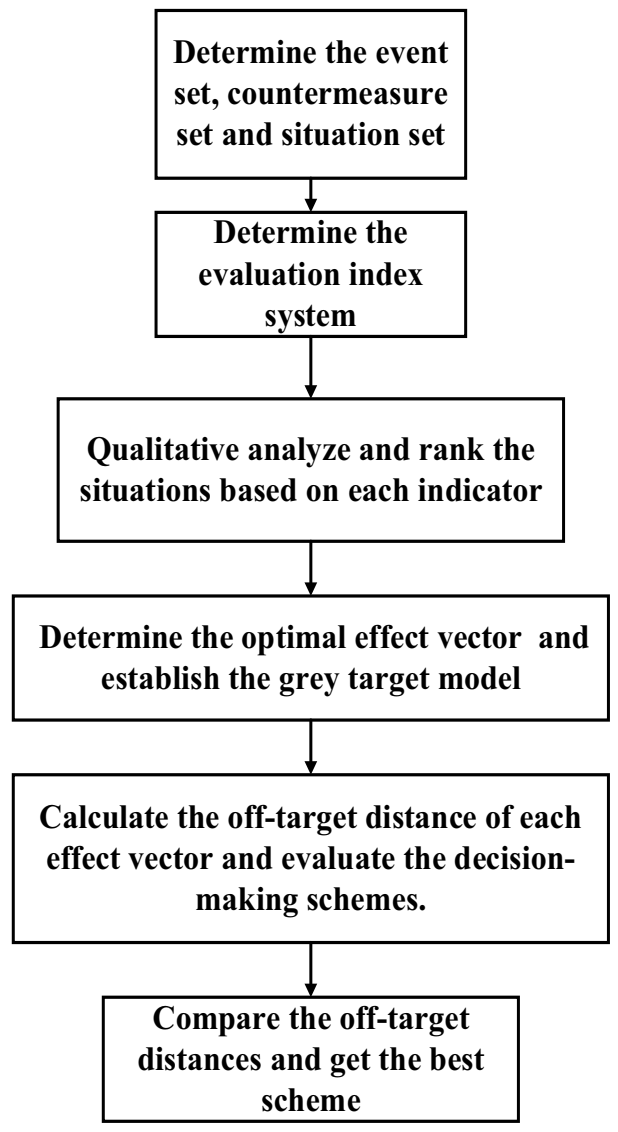

Figure 1. Flow chart of grey target decision algorithm

\subsection{Decision-making Model}

Different sections of existing high-speed railway run at different speeds according to the limitations of the line, so the speed-up target values are different in sections. In order to maintain the independence of each situation in the problem, the event set is $A=\left\{a_{1}\right.$ : speed-up $\}$, and the countermeasure set $B=\left\{b_{1}, b_{2}, \ldots b_{m}\right\}$ is the set of speed-up schemes in different sections. 
Any combination of elements in $A$ and $B$ constitutes the situation $s_{i j}$ with respect to $\mathrm{n}$ evaluation indicators.According to the analysis above, for the decision-making problem with s objectives, the optimal effect vector is $\boldsymbol{r}_{0}=\left(r_{0}{ }^{(1)}, r_{0}{ }^{(2)}, \ldots, r_{0}{ }^{(s)}\right)$ after sorting.

$$
R=\left\{\left(r^{(1)}, r^{(2)}, \ldots, r^{(s)}\right) \mid\left(r^{(1)}-r_{0}^{(1)}\right)^{2}+\left(r^{(2)}-r_{0}^{(2)}\right)^{2}+\ldots+\left(r^{(s)}-r_{0}^{(s)}\right)^{2} \leq \mathrm{R}^{2}\right\}
$$

is the S-dimensional spherical grey target with $\boldsymbol{r}_{0}$ as the target center and $\mathrm{R}$ as radius.

For the scheme $\boldsymbol{r}_{\boldsymbol{1}}=\left(r_{1}^{(1)}, r_{1}^{(2)}, \ldots, r_{1}^{(s)}\right) \in R$, $\left|\boldsymbol{r}_{1}-\boldsymbol{r}_{0}\right|=\sqrt{\left(r^{(1)}-r_{0}^{(1)}\right)^{2}+\left(r^{(2)}-r_{0}^{(2)}\right)^{2}+\ldots+\left(r^{(s)}-r_{0}^{(s)}\right)^{2}}$ is called the off-target distance of the vector $\boldsymbol{r}_{1}$.

For different situations $s_{i j}$ and $s_{h j}$, the corresponding effect vectors are $\boldsymbol{u}_{i j}=\left(u_{i j}{ }^{(1)}, u_{i j}{ }^{(2)}, \ldots, u_{i j}{ }^{(s)}\right)$ and $\boldsymbol{u}_{h j}=\left(u_{h j}{ }^{(1)}, u_{h j}{ }^{(2)}, \ldots, u_{h j}{ }^{(s)}\right)$.

If the off-target distance $\left|\boldsymbol{u}_{\boldsymbol{i j}}-\boldsymbol{r}_{0}\right| \geq\left|\boldsymbol{u}_{\boldsymbol{h j}}-\boldsymbol{r}_{0}\right|$, the situation $s_{h j}$ is better than $s_{i j}$.If the equal sign holds, the two situations are considered equivalent [13].

\section{Application Cases}

A high-speed railway line contains four different sections with designed speed of $200 \mathrm{~km} / \mathrm{h}$ and $250 \mathrm{~km} / \mathrm{h}$ respectively. It is planned to raise the speed of different sections of this line, as shown in Table 1.

Table 1. Target of sectional speed increase

\begin{tabular}{ccl}
\hline $\begin{array}{c}\text { Original speed of section } \\
(\mathbf{k m} / \mathbf{h})\end{array}$ & $\begin{array}{c}\text { Proposed lifting speed } \\
(\mathbf{k m} / \mathbf{h})\end{array}$ & Corresponding speed-up scheme \\
\hline Section 1, 4:200 & 250 & $\mathrm{~b}_{1:}$ speed up of section 1 and 4 \\
Section 2: 250 & 300 & $\mathrm{~b}_{2}:$ speed up of section 2, section 1 and 4 \\
Section 3:250 & 280 & $\mathrm{~b}_{3}:$ speed up of section 3, section 1 and 4 \\
& & b4: speed up of the whole line \\
\hline
\end{tabular}

In this paper, the event set is $A=\left\{a_{1}\right.$ : speed-up $\}$. In order to study conveniently and keep the independence of each situation in the problem, the same-speed sections are considered together. The corresponding countermeasure set is as follows: $B=\left\{b_{1}\right.$ : speed up of sections 1 and $4 ; b_{2}$ : speed up of sections 2,1 and $4 ; b_{3}$ : speed up of sections 3, 1 and 4; b4: speed up of the whole line $\}$. The corresponding situations are as follows: $s_{11}=\left(a_{1}, b_{1}\right)=$ (speed-up; speed up of section 1 and 4$)$; $s_{12}=\left(a_{1}, b_{2}\right)$ (speed-up; speed up of section 2 , section 1 and 4$) ; s_{13}=\left(a_{1}, b_{3}\right)=$ (speed-up; speed up of section 3, section 1 and 4$) ; s_{14}=\left(a_{1}, b_{4}\right)=$ (speed-up; speed up of the whole line). The situations remain relatively independent.

The evaluation indexes are efficiency, cost and influence. Because the environment of different sections of high-speed railway is different and the influence factors are 
complex, it is difficult to obtain quantitative evaluation for corresponding indexes. In the decision-making of initial scheme, the situation under each target is analyzed qualitatively. Based on the evaluation objectives, the corresponding situations are qualitatively analyzed and sorted, as shown in Table 2.

Table 2. Qualitative analysis on index performance of speed-up scheme

\begin{tabular}{ccccc}
\hline scheme & $\mathbf{s}_{\mathbf{1 1}}$ & $\mathbf{s}_{\mathbf{1 2}}$ & $\mathbf{s}_{\mathbf{1 3}}$ & $\mathbf{s}_{\mathbf{1 4}}$ \\
\hline efficiency & poor & excellent & medium & optimal \\
cost & optimal & excellent & medium & poor \\
influence & optimal & excellent & medium & poor \\
\hline
\end{tabular}

The values 1,2,3,4 are assigned to qualitative evaluation 'Optimal, excellent, medium and poor', and the effect vectors of situation $s_{i j}$ are as follows:

$$
\begin{aligned}
& \boldsymbol{u}_{11}=\left(u_{11}{ }^{(1)}, u_{11}{ }^{(2)}, u_{11}{ }^{(3)}\right)=(4,1,1) \\
& \boldsymbol{u}_{12}=\left(u_{12}{ }^{(1)}, u_{12}{ }^{(2)}, u_{12}{ }^{(3)}\right)=(2,2,2) \\
& \boldsymbol{u}_{13}=\left(u_{13}{ }^{(1)}, u_{13}{ }^{(2)}, u_{13}{ }^{(3)}\right)=(3,3,3) \\
& \boldsymbol{u}_{14}=\left(u_{14}{ }^{(1)}, u_{14}{ }^{(2)}, u_{14}{ }^{(3)}\right)=(1,4,4)
\end{aligned}
$$

Therefore, the optimal vector corresponding to the spherical grey target is $\boldsymbol{r}_{0}=(1,1,1)$.

When the radius of the grey target is 5 , the off-target distances of the four schemes are obtained as follows:

$$
\begin{aligned}
& \left|\boldsymbol{u}_{11}-\boldsymbol{r}_{0}\right|=\sqrt{\left(u_{11}{ }^{(1)}-r_{0}{ }^{(1)}\right)^{2}+\left(u_{11}{ }^{(2)}-r_{0}{ }^{(2)}\right)^{2}+\left(u_{11}{ }^{(3)}-r_{0}^{(3)}\right)^{2}}=3 \\
& \left|\boldsymbol{u}_{12}-\boldsymbol{r}_{0}\right|=\sqrt{\left(u_{12}{ }^{(1)}-r_{0}^{(1)}\right)^{2}+\left(u_{12}{ }^{(2)}-r_{0}{ }^{(2)}\right)^{2}+\left(u_{12}{ }^{(3)}-r_{0}^{(3)}\right)^{2}}=\sqrt{3} \\
& \left|\boldsymbol{u}_{13}-\boldsymbol{r}_{0}\right|=\sqrt{\left(u_{13}{ }^{(1)}-r_{0}^{(1)}\right)^{2}+\left(u_{13}{ }^{(2)}-r_{0}^{(2)}\right)^{2}+\left(u_{13}{ }^{(3)}-r_{0}^{(3)}\right)^{2}}=2 \sqrt{3} \\
& \left|\boldsymbol{u}_{14}-\boldsymbol{r}_{0}\right|=\sqrt{\left(u_{14}{ }^{(1)}-r_{0}^{(1)}\right)^{2}+\left(u_{14}{ }^{(2)}-r_{0}{ }^{(2)}\right)^{2}+\left(u_{14}{ }^{(3)}-r_{0}^{(3)}\right)^{2}}=3 \sqrt{2}
\end{aligned}
$$

The off-target distance of each scheme is shown in Figure 2. 


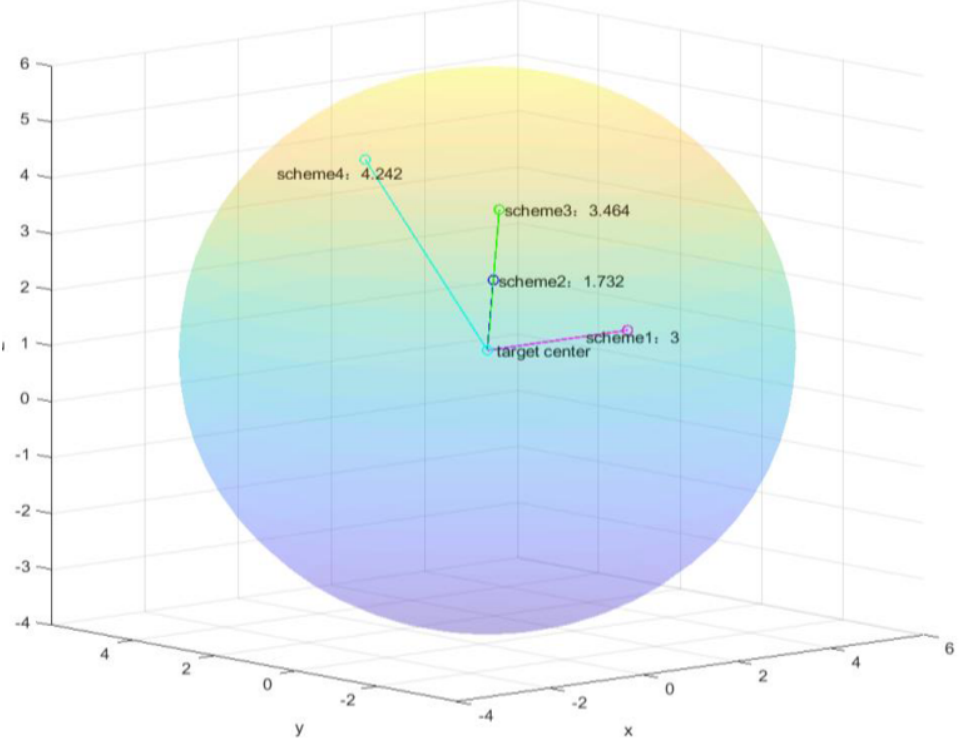

Figure 2. The off-target distance of each scheme

It can be seen from Figure 2 that all four schemes are optional in the selected spherical grey target. By comparing the off-target distance, it can be concluded that the off-target distance of scheme 2, 1.732, is minimum. So the corresponding speed-up scheme of section 1, 4 and section 2 should be selected as the optimal scheme for the high-speed railway.

\section{Epilogue}

Based on the analysis above, due to the difference of the operation speed in different sections of high-speed railway and the uncertainty of the implementation of the speed-raising project, it is necessary to study the speed-raising of high-speed railway piecewise, and its influencing factors are mainly efficiency, cost and influence. Compared with other methods, the grey target decision model used in this paper can qualitatively rank schemes to obtain the optimal results without quantitative data.Based on the transformation of Euclidean space index measure, the off-target distance of each scheme in spherical grey target is compared, and the corresponding scheme of minimum off-target distance is the optimal decision-making scheme. The rationality of the model and the effectiveness of the method are verified by an example.

\section{Acknowledgement}

Research is supported by the fund from China Academy of Railway Sciences (Number: K2019G043) 


\section{Reference}

[1] Xiao X, Fu Y, Zhang LY. Analysis of scale economy before and after China's railway speed increase. Journal of Dalian Jiaotong University, 2010,31 (02): 104-107 + 112

[2] Ministry of Railways of the people's Republic of China. Main technical policies for railways. Beijing: China Railway Press, 1983-2013

[3] Ren XJ. The driving role of speed up strategy on the development of China's railway transportation. Management and technology of small and medium-sized enterprises (Journal of midmonth), 2019 (02): $39-40$

[4] Shi ZK, Shao J, Pu ZN. Transportation infrastructure improvement and productivity growth: evidence from railway speed increase. World economy, 2018,41 (06): 127-151

[5] Zhou H, Yu JL. Railway speed increase, accessibility and urban economic growth. Economic review, 2013 (01): $52-59+70$

[6]Yang YH, Wang YL, Wang NM. Research on Fuzzy equilibrium optimization of construction period cost and quality of engineering project. Theory and practice of systems engineering, 2006,26 (7): 112-117

[7] Ge SY, Huo CJ, Liu H. Transformation of overhead line into ground based on improved fuzzy set gravity center comprehensive decision method. Journal of Tianjin University, 2007,40 (2): 195-201.

[8] Song JP, Dong DW, Gao GA. Research on scheme decision model based on analytic hierarchy process and grey relational degree Journal of Southwest Jiaotong University, 2002,37 (4): 463-466

[9] Liang B, Qin B, Sun WJ, etc. Application of intelligent weighted grey target decision model in risk assessment of coal and gas outburst. Journal of coal industry, 2013,38 (9): 1611-1615

[10] Hua C. Multi objective optimization analysis and decision model research of railway line scheme based on grey theory . Railway survey, 2020,46 (4): 88-92

[11] Wang L. Research on the influence of speed increase on railway transportation supply.Southwest Jiaotong University, 2004

[12] Wang ZX, Dang YG, Yang H. Improved multi-objective grey target decision making method. Systems engineering and electronic technology, 2009,31 (11): 2634-2636

[13] Wang KD, Zhou XZ. New edition of theory and method of operational planning and decision making.Beijing: Tsinghua University Press, 2010 